\title{
Electromagnetic Wave Propagation in Waveguide Loaded by Split Ring Resonator of Negative Permeability
}

\author{
Abd El Moneim M. Alaa, Mostafa El Said, Samir F. Mahmoud \\ Electronics and Communications Department, Faculty of Engineering, Cairo University, Cairo, Egypt \\ Email: abiza2@msn.com
}

How to cite this paper: Alaa, A.E.M.M., El Said, M. and Mahmoud, S.F. (2017) Electromagnetic Wave Propagation in Waveguide Loaded by Split Ring Resonator of Negative Permeability. Journal of Electromagnetic Analysis and Applications, 9, 113-121.

https://doi.org/10.4236/jemaa.2017.98010

Received: August 12, 2017

Accepted: August 28, 2017

Published: August 31, 2017

Copyright $\odot 2017$ by authors and Scientific Research Publishing Inc. This work is licensed under the Creative Commons Attribution International License (CC BY 4.0).

http://creativecommons.org/licenses/by/4.0/

\begin{abstract}
This paper aims to study and analyze the electromagnetic propagation in media with negative transverse permeability and how this leads into some physical phenomena such as the appearance of backward waves and the propagation below cutoff. This study is done through the use of metamaterials of split ring resonators. It is shown that the waveguide dimensions needed to transmit a certain frequency band, can be miniaturized to half its dimension. The analytical determination of the propagation inside a waveguide in the presence of two slabs with dielectric permittivity and negative transverse permeability is derived. Finally it is shown by simulation, how to obtain a backward wave with lower loss than reported earlier in the literature.
\end{abstract}

\section{Keywords}

Negative Transverse Permeability, Metamaterials, Backward Waves,

Miniaturization of Waveguide, Propagation Below Cutoff

\section{Introduction}

Rectangular waveguides are required for most of applications in microwaves. It can be used as a basic guided structure in radar application. The important application of the waveguide is to radiate element in multi-frequency interlaced antenna arrays. There are several methods to reduce the size of waveguide. One of the most important methods is metamaterial that it is used to reduce the size of the waveguide and to obtain the desired resonant frequency bands. At a particular frequency, metamaterials exhibit both negative permittivity and permeability [1] [2] [3] [4] [5].

In 1968 Veselago [1] analyzed electromagnetic wave propagation through 
media with negative electric permittivity $\varepsilon$ and magnetic permeability $\mu$. The fields and the wave propagation form a left-handed system in these materials, but the nonexistence of transparent left-handed media in nature made Veselago's results just a theory. Recently, Smith et al. [2] [3] have demonstrated microwave propagation through an artificial left-handed medium (metamaterial).

Several names and terminologies have been suggested for metamaterials with negative permittivity and permeability, such as "left-handed", "backward-wave media" and "double-negative". Nowadays, many researchers are studying various aspects of this class of metamaterials, and several ideas and suggestions for future applications have been proposed [4] [5] [6].

The edge coupled split ring resonators (EC-SRR) are proposed by Pendry et al. [7] and experimentally tested by Smith et al. [2] and Marque's, R., et al. [8] (Figure 1(b)). They are composed of electrically small resonant rings, which show a very high diamagnetic susceptibility above and around its resonance frequency.

The magnetic and electric dipole of the EC-SRR can be expressed by [9]:

$$
\begin{aligned}
& m_{x}=\alpha_{x x}^{m m} B_{x}^{e x t}-\alpha_{y x}^{e m} E_{y}^{e x t} \\
& P_{y}=\alpha_{y y}^{e e} E_{y}^{e x t}+\alpha_{x y}^{e m} B_{x}^{e x t}
\end{aligned}
$$

The Bianistropy terms $\alpha_{y x}^{e m} \& \alpha_{x y}^{m e}$ occurred due to the fact that SRR does not act only as a magnetic dipole [9] [10], but also as an electric dipole.

Avoiding Bianistropy of the EC-SRR by a modification to the Broadside-coupled split ring resonator (Figure $1(\mathrm{a})$ ), the BC-SRR has inversion symmetry with regard to the center of both rings. Therefore the cross-polarizability terms must vanish. So the Bianistropy terms $\alpha_{y x}^{e m} \& \alpha_{x y}^{m e}$ are equal to zero, and the magnetic and electric dipole can be written as [10] [11]:

$$
\begin{gathered}
m_{x}=\alpha_{x x}^{m m} B_{x}^{e x t} \\
P_{y}=\alpha_{y y}^{e e} E_{y}^{e x t}
\end{gathered}
$$

The SRR loaded waveguide supports the propagation of backward waves below the cut-off frequency of the air-filled waveguide [12] [13] [14] [15] [16]. It provides the miniaturization of waveguide. Hrabar et al. [13], showed that

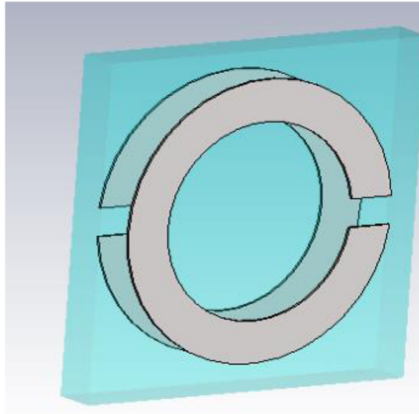

(a)
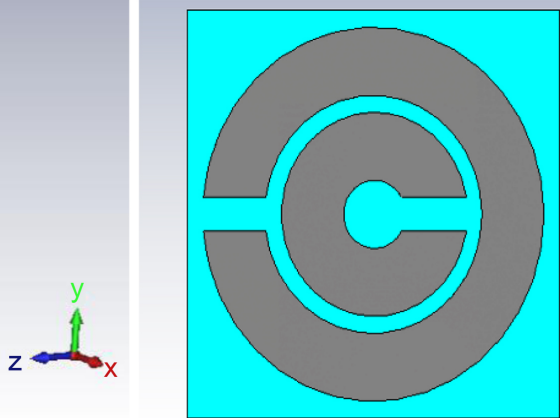

(b)

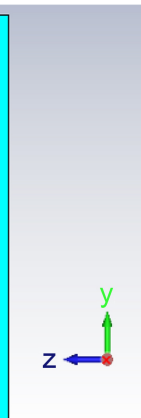

Figure 1. (a) (BC-SRR); (b) (EC-SRR). 
backward propagation occurs when the longitudinal permeability is positive and the transversal permeability is negative, but it is noticed that a large insertion loss of almost $25 \mathrm{~dB}$ occurs in the $\mathrm{S}_{12}$ measurement results in the backward wave, and also it has a very narrow bandwidth.

The aim of this paper is to increase the bandwidth and decrease the losses of the backward wave, through maximizing the negative magnetic permeability.

\section{The Proposed Design}

From the proposed configuration of Schelkunoff's [17], the magnetic polarizability of a closed metallic loop of radius $r$ loaded by a capacitor is expressed as:

$$
\alpha_{x x}^{m m}=\frac{\pi^{2} r^{4}}{L}\left(\frac{\omega_{o}^{2}}{\omega^{2}}-1\right)^{-1}
$$

where $\omega_{0}$ is the resonant frequency of the LC circuit formed by the loop and the capacitor. It is shown from Equation (5) that, just above the frequency of resonance, the polarizability becomes negative and very large. Therefore, it is expected that a regular array of capacitive loaded metallic loops will show a negative magnetic permeability just above the frequency of resonance of the loops [11]. According to schelkunoff's [17] and Marque's [11], if two or more split rings resonator are formed in a regular array, it will show a large negative magnetic permeability just above the resonance frequency of the rings.

By separating the two rings each on a single substrate and with opposite slots as shown in Figure 2, a regular array of capacitive loading ring "the gap capacitance of the slot and the surface capacitance" will show a large negative magnetic permeability in the direction of the magnetic dipole. In addition to the advantage of avoiding the bianistropy, where the electric polarization of the upper half side $(y>0)$ must equal to the opposite electric polarization of the lower half side $(y<0)$ of the rings, so the design is not bianisotropic. The magnetic dipole of the proposed design resulting from the regular array of the two rings, can be expressed by:

$$
\sum_{i=1}^{n} M_{x_{i}}=\alpha_{x x}^{m m} B_{x}^{e x t}
$$

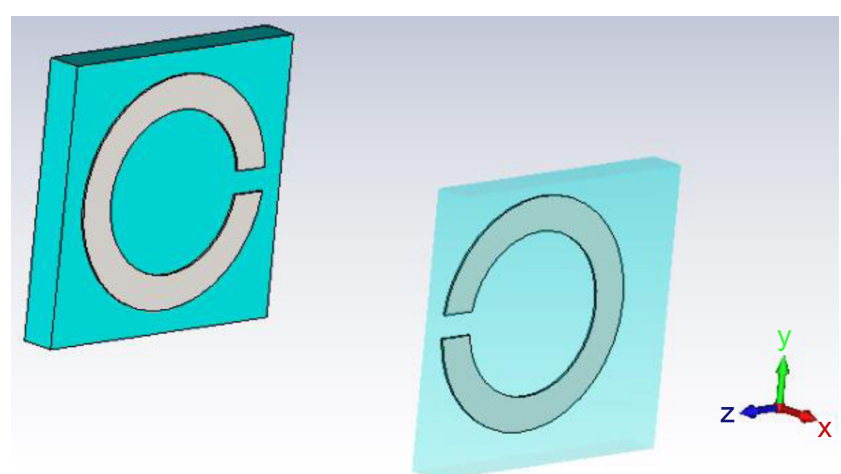

Figure 2. Two separated substrates with opposite single split ring resonator. 
where, $n$ is the number of rings in $\mathrm{x}$-direction.

\section{Theoretical Analysis}

A rectangular waveguide is loaded by two slabs each of $\varepsilon_{r}$ and negative transverse permeability $\mu_{t r}$ due to the presence of split ring resonator, the two slabs are located in the waveguide as shown in Figure 3.

The electric field $E_{y}$ in the different regions is given as:

In air

$$
E_{y}=\left\{\begin{array}{lc}
A \sin \left(k_{x o}(x)\right) \mathrm{e}^{-i \beta z}, & 0 \leq x \leq m \\
B \cos \left(k_{x o}\left(\frac{a}{2}-x\right)\right) \mathrm{e}^{-i \beta z}, & (d+m) \leq x \leq(a-m-d) \\
C \sin \left(k_{x o}(a-x)\right) \mathrm{e}^{-i \beta z}, & (a-m) \leq x \leq a
\end{array}\right.
$$

In slab

$$
E_{y}= \begin{cases}{\left[\frac{A \sin \left(k_{x o}(m)\right)}{\sin \left(k_{x}(m)\right)}\right] \sin \left(k_{x}(x)\right) \mathrm{e}^{-i \beta z},} & m \leq x \leq(d+m) \\ B \cos \left(k_{x o}\left(m+d-\frac{a}{2}\right)\right)\left[\frac{\sin \left(k_{x}(a-x)\right)}{\sin \left(k_{x}(m+d)\right)}\right] \mathrm{e}^{-i \beta z}, & (a-m-d) \leq x \leq(a-m)\end{cases}
$$

While, from the basic of Electromagnetic propagation inside a waveguide

$$
E_{x}=0, \quad E_{z}=0, \quad H_{y}=0
$$

The magnetic field $H$ is obtained from the Maxwell's equation:

$$
\nabla X E=-j \omega[\mu] H
$$

And, the magnetic permeability tensor is:

$$
\mu=\mu_{o}\left[\begin{array}{ccc}
\mu_{t r} & 0 & 0 \\
0 & 1 & 0 \\
0 & 0 & 1
\end{array}\right]
$$

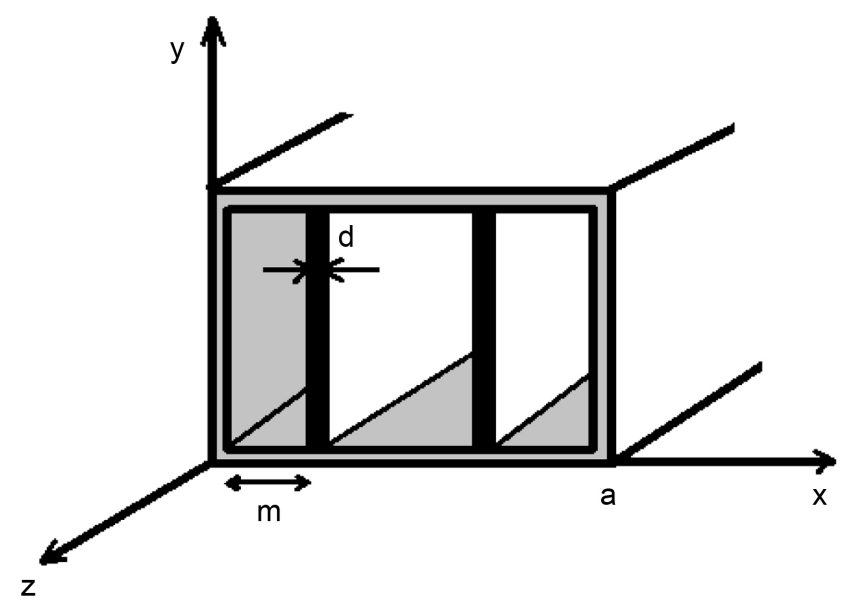

Figure 3. Two slabs with negative transverse permeability located in a waveguide. 
We can assume a magnetic wall in the middle of the waveguide as shown in Figure 4, while the walls of the waveguide are electric walls.

Then, the wave equation in air region is applied to get:

$$
k_{X o}^{2}+\beta^{2}=k_{o}^{2}
$$

And the boundary condition is applied at $x=m+d$, we get:

$$
\frac{\tan \left[k_{X}(m+d)\right]}{k_{X}}-\frac{\cot \left[k_{x o}\left(\frac{a}{2}-m-d\right)\right]}{k_{X o}}=0
$$

And from Maxwell's equation:

$$
\nabla X H=j \omega \varepsilon E
$$

Applying wave equation in slab region to get:

$$
k_{x}^{2}+\frac{\beta^{2}}{\mu_{r}}=\omega^{2} \mu_{o} \varepsilon_{o} \varepsilon_{r}
$$

From Equation (11), the wave propagation factor can be expressed as:

$$
\beta=\sqrt{\mu_{r} \varepsilon_{r}\left(k_{o}^{2}-\frac{k_{x}^{2}}{\varepsilon_{r}}\right)}
$$

We define the cutoff frequency of the partially filled waveguide with metamaterial as $f_{c p}$.

It can be shown that:

$$
f_{c p}>f_{c}=\frac{f_{c o}}{\sqrt{\varepsilon_{r}}}
$$

where $f_{c o}$ is the cutoff frequency of air-filled waveguide, therefore:

$$
\beta=k_{o} \sqrt{\mu_{r} \varepsilon_{r}\left(1-\left(\frac{f_{c p}}{f}\right)^{2}\right)}
$$

In Equation (13), if $\varepsilon_{r}$ and $\mu_{t r}$ are positive, propagation above cutoff occurs.

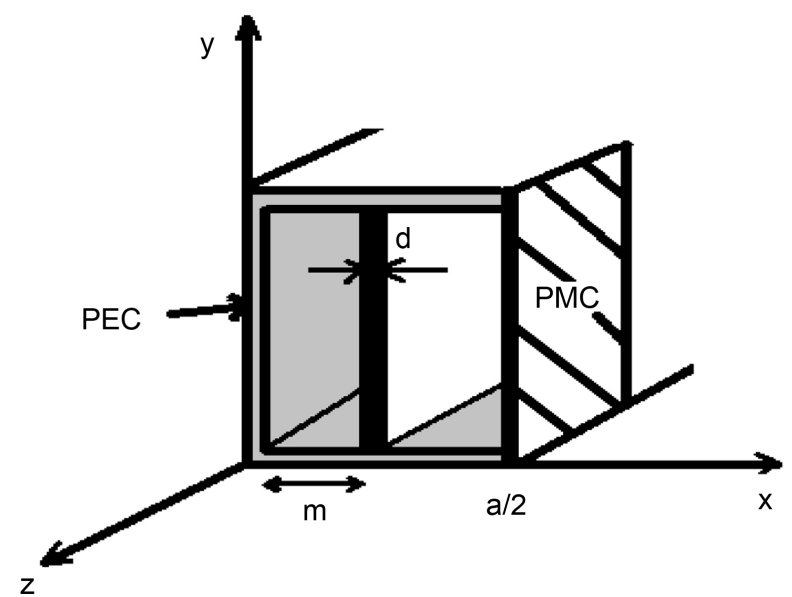

Figure 4. Equivalent waveguide. 
Also if $\varepsilon_{r}$ is negative, $\mu_{t r}$ must be negative for propagation to occur. The interesting case is when the $\mu_{t r}$ is negative and $f_{c p}>f$, where propagation below cut off occurs.

A simulation for the propagation constant $\beta$ versus frequency is shown in Figure 5 at different values of negative transverse permeability $\mu_{t r}$ and $(m=$ $2.6 \mathrm{~mm}, a=12 \mathrm{~mm}$ ).

It seems that at a certain frequency, the propagation constant increased as the negative permeability increased. Meanwhile, at same negative permeability, the propagation constant is decreasing with the increasing of the frequency.

\section{Results}

We have designed two rings of opposite slots direction at resonance frequency $f_{o}=8.25 \mathrm{GHz}$ with the following parameters, $R_{i}=1.75 \mathrm{~mm}, R_{o}=2.5 \mathrm{~mm}$ and slot width $0.5 \mathrm{~mm}$, and etched on copper cladding substrate with thickness $0.35 \mathrm{~mm}$, copper thickness $0.02 \mathrm{~mm}$, and dielectric permittivity $\varepsilon_{r}=2.6$. By using CST MW Studio, the simulated result of S12 is shown in Figure 6. The 10 $\mathrm{db}$ bandwidth of $\mathrm{S} 12$ extends from 8.1 to $8.5 \mathrm{GHz}$.

Two slabs each of ten SRRs with opposite slots direction are inserted symmetrically along the center of waveguide of dimensions $(12 \mathrm{~mm} \times 12 \mathrm{~mm})$, the

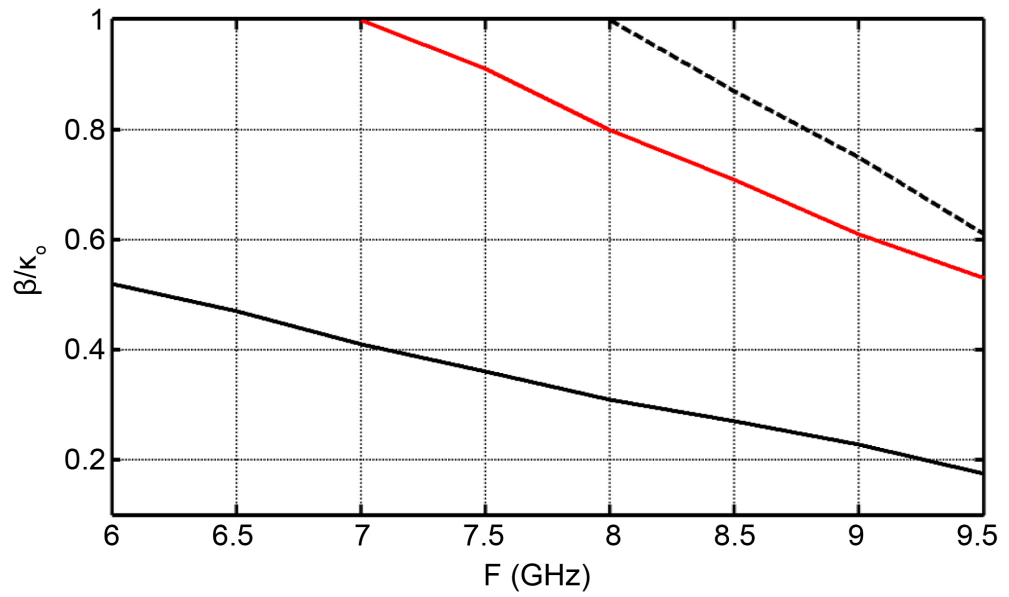

Figure 5. The propagation constant $\beta / k_{o}$ versus resonance frequency $f_{o}$.

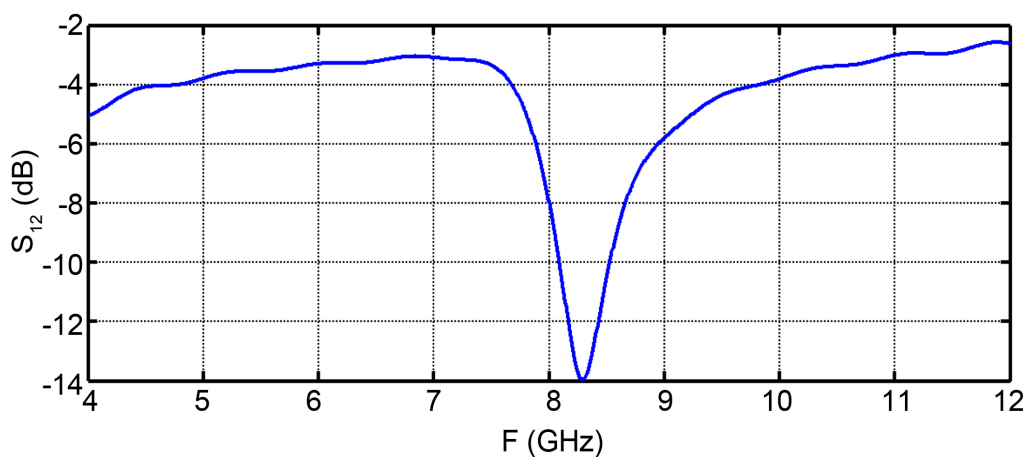

Figure 6. The result of $S_{12}$ for a Single split ring resonator. 
lattice constant is $6 \mathrm{~mm}$ and distance between two slabs $=6.5 \mathrm{~mm}$. By using CST MW Studio, the simulated results $S_{12}$ are shown in Figure 7.

In Figure 7, when a regular array of capacitive loaded rings are inserted in a waveguide, a large negative magnetic permeability in the direction of the magnetic dipole at $\left(f_{o}=8.7 \mathrm{GHz}\right)$ occurs just above the frequency of resonance of the rings $(8.25 \mathrm{GHz})$. The $\mathrm{S} 12$ reached $0 \mathrm{db}$ at no losses, while by adding losses of the substrate and the copper clad $\left(\sigma=2 \times 10^{7} \mathrm{~s} / \mathrm{m}\right)$, the $S_{12}$ reaches $-4 \mathrm{db}$, while in [14] the $S_{12}$ reached $-10 \mathrm{db}$ in lossless case and $-28 \mathrm{db}$ in lossy case.

The result of the $3 \mathrm{db}$ bandwidth for the backward wave of Figure 7 is shown in Figure 8.

In Figure 8, it is shown that a bandwidth of $95 \mathrm{MHz}$ has been achieved, which is wider than the bandwidth reported in the literature [12], where it was about $70 \mathrm{MHz}$. This means that, the bandwidth of the proposed design has increased by $30 \%$ relative to that reported in literature [14] [15].

By changing the following parameters $\left(R_{i}, R_{o}, \mathrm{~m} / \mathrm{a}\right)$ and applying simulation program CST MW studio, we have got the influences of these parameters on the resonant frequency $f_{o}$ as shown in Table 1 and plotted in Figure 9.

\section{Conclusion}

A waveguide filled with negative permeability metamaterial SRR of resonant

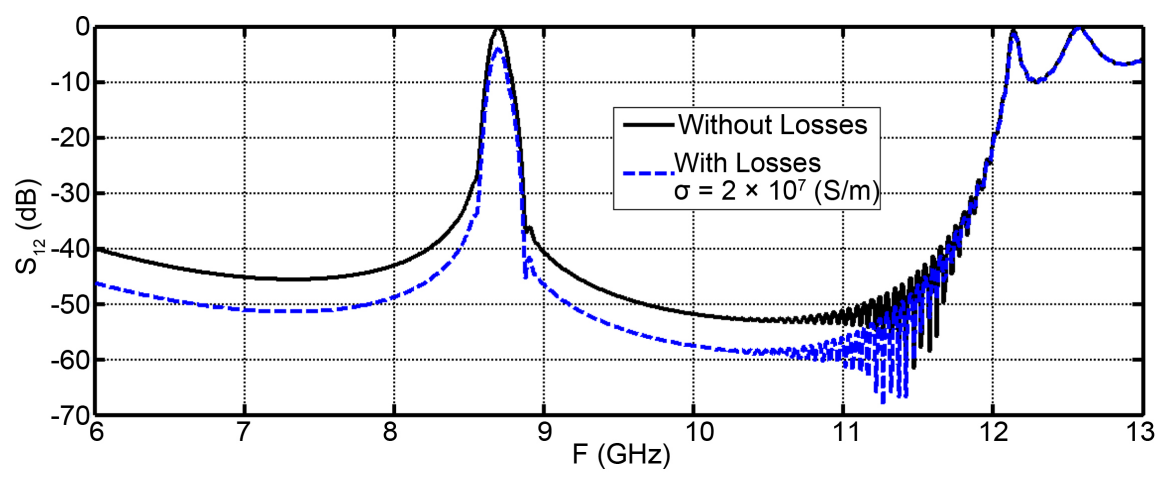

Figure 7. The result of $S_{12}$, the solid line of a waveguide filled with the propose design and the dotted line with adding losses to $\mathrm{Cu}$ Clad and substrate with $\sigma=2 \times 10^{7} \mathrm{~s} / \mathrm{m}$.

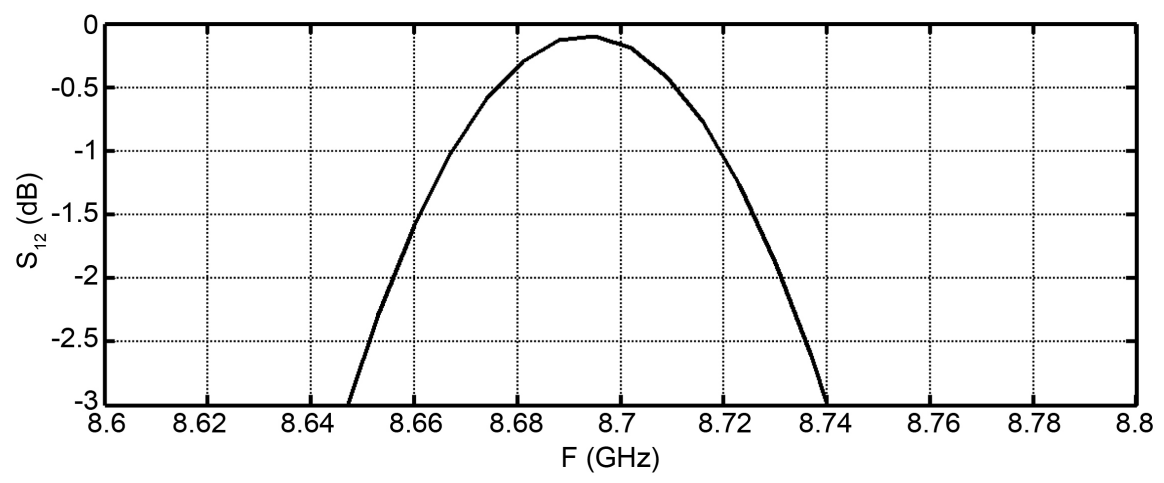

Figure 8. The result of $3 \mathrm{db}$ of the backward wave for the proposed design. 
Table 1. Parametric study of different resonant frequency.

\begin{tabular}{ccccc}
\hline & $R_{i}$ & $R_{o}$ & $m / a$ & $f_{o}$ \\
\hline $\mathrm{a}$ & $2 \mathrm{~mm}$ & $2.5 \mathrm{~mm}$ & 0.225 & $8.3 \mathrm{GHz}$ \\
$\mathrm{b}$ & $1.75 \mathrm{~mm}$ & $2.5 \mathrm{~mm}$ & 0.2 & $8.75 \mathrm{GHz}$ \\
$\mathrm{c}$ & $1.7 \mathrm{~mm}$ & $2 \mathrm{~mm}$ & 0.175 & $9.2 \mathrm{GHz}$ \\
$\mathrm{d}$ & $1.7 \mathrm{~mm}$ & $2.4 \mathrm{~mm}$ & 0.14 & $10 \mathrm{GHz}$ \\
$\mathrm{e}$ & $1.5 \mathrm{~mm}$ & $2.4 \mathrm{~mm}$ & 0.1 & $10.5 \mathrm{GHz}$ \\
\hline
\end{tabular}

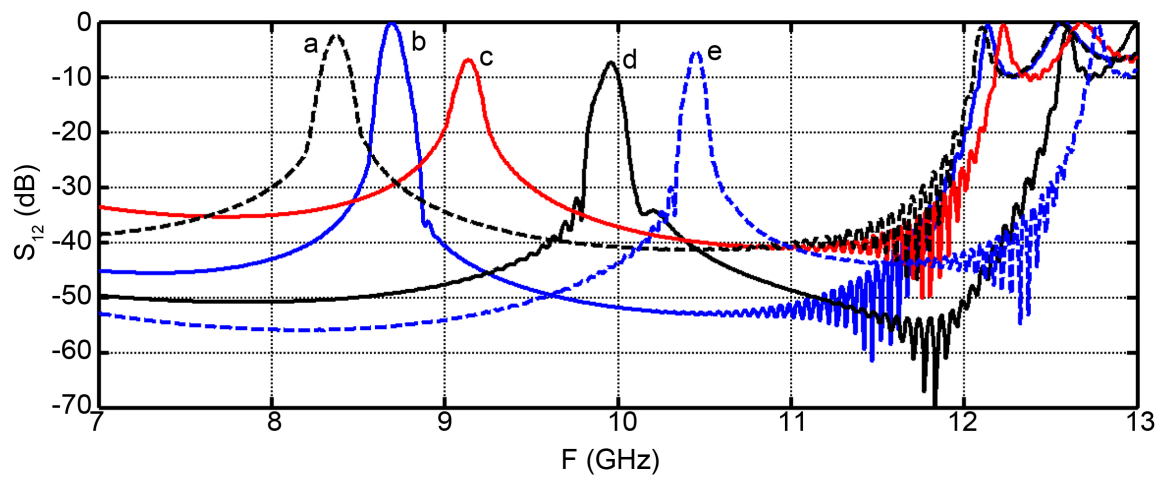

Figure 9. The result of $S_{12}$ for each case in Table 1 .

frequency $f_{o}=7.8 \mathrm{GHz}$ has been analyzed theoretically and simulated by CST MW Studio, and is shown to support a backward propagation below cutoff when the transverse permeability is negative. We have added two slabs symmetrically in waveguide to increase magnetic dipole of the rings. The transverse width of the waveguide can just be miniaturized to smaller than the half of an empty waveguide needed to propagate this frequency. As a result, the losses of the backward wave are decreased to $0 \mathrm{db}$ and the bandwidth becomes wider than that reported in the literature by about $30 \%$.

\section{References}

[1] Veselago, V.G. (1968) The Electrodynamics of Substances with Simultaneously Negative Values of $\varepsilon$ and $\mu$. Soviet Physics Uspekhi, 10, 509. https://doi.org/10.1070/PU1968v010n04ABEH003699

[2] Smith, D.R., Padilla, W.J., Vier, D.C., Nemat-Nasser, S.C. and Schultz, S. (2000) Composite Medium with Simultaneously Negative Permeability and Permittivity. Physical Review Letters, 84, 4184. https://doi.org/10.1103/PhysRevLett.84.4184

[3] Shelby, R.A., Smith, D.R., Nemat-Nasser, S.C. and Schultz, S. (2001) Microwave Transmission through a Two-Dimensional, Isotropic, Left-Handed Metamaterial. Applied Physics Letters, 78, 489-491.

[4] Engheta, N. and Ziolkowski, R.W. (2006) Metamaterials: Physics and Engineering Explorations. IEEE Press, The Institute of Electrical and Electronics Engineers, Inc.

[5] Indhumathi, G., Karthika, G.S. and Muthulakshmi, S. (2017) Metamaterial Triangular Split Ring Resonator Antenna for Wireless Applications. SSRG International Journal of Electronics and Communication Engineering, (ICRTECITA-2017), Special Issue, March 2017. 
[6] Bage, A. and Das, S. (2016) A Compact, Wideband Waveguide Bandpass Filter Using Complementary Loaded Split Ring Resonators. Progress in Electromagnetics Research C, 64, 51-59. https://doi.org/10.2528/PIERC16040102

[7] Pendry, J.B., Holden, A.J., Robbins, D.J. and Stewart, W.J. (1999) IEEE Transactions on Microwave Theory and Techniques. Magnetism from Conductors and Enhanced Nonlinear Phenomena, 47.

[8] Marqués, R., Martel, J., Mesa, F. and Medina, F. (2002) Left-Handed-Media Simulation and Transmission of EM Waves in Subwavelength Split-Ring-Resonator-Loaded Metallic Waveguides. Physical Review Letters, 89, 183901-1-183901-4.

[9] Marqués, R., Mesa, F., Martel, J. and Medina, F. (2003) Comparative Analysis of Edge- and Broadside-Coupled Split Ring Resonators for Metamaterial Design-Theory and Experiments. Transactions on Antennas and Propagation, 51, 2572-2581.

[10] Marqués, R., Medina, F. and Rachid Rafii-El-Idrissi, R. (2002) Role of Bianisotropy in Negative Permeability and Left-Handed Metamaterials. Physical Review B, 65, 144440-1-14440-6.

[11] Marqués, R., Martin, F. and Sorolla, M. (2008) Metamaterials with Negative Parameters Theory, Design, and Microwave Applications. John Willey and Sons.

[12] Hrabar, S. (2006) Basic Radiation Properties of Waveguides Filled with Uniaxial Single Negative Metamaterials. Microwave and Optical Technology Letters, 48, 2587-2591. https://doi.org/10.1002/mop.21993

[13] Sipus, Z. (2004) Miniaturization of Rectangular Waveguide Using Uniaxial Negative Permeability Meta-Material. Proceedings of the 12 th IEEE Mediterranean Electrotechnical Conference, 12-15 May 2004, 495-498.

[14] Meng, F., Wu, Q., Erni, D. and And Li, L. (2011) Controllable Metamaterial-Loaded Waveguides Supporting Backward and Forward Waves. Transactions on Antennas and Propagation, 59, 3400-3411. https://doi.org/10.1109/TAP.2011.2161540

[15] Hrabar, S., Bartolic, J. and Sipus, Z. (2005) Waveguide Miniaturization Using Uniaxial Negative Permeability Metamaterial. IEEE Transactions on Antennas and Propagation, 53, 110-119.

[16] Mohamed, K. and Nidal, A. (2013) Rectangular Waveguide Radiator Miniaturization using Electromagnetic Infinity-Shaped Metamaterial Resonator. IUG Journal of Natural and Engineering Studies, 21, 69-82.

[17] Shelkunoff, S.A. and Friis Antennas, H.T. (1966) Theory and Practice. 3rd Edition, Wiley, New York. 
Submit or recommend next manuscript to SCIRP and we will provide best service for you:

Accepting pre-submission inquiries through Email, Facebook, LinkedIn, Twitter, etc. A wide selection of journals (inclusive of 9 subjects, more than 200 journals)

Providing 24-hour high-quality service

User-friendly online submission system

Fair and swift peer-review system

Efficient typesetting and proofreading procedure

Display of the result of downloads and visits, as well as the number of cited articles Maximum dissemination of your research work

Submit your manuscript at: http://papersubmission.scirp.org/

Or contact jemaa@scirp.org 